Pacific Journal of Mathematics

TOPOLOGICAL EXTENSIONS OF PRODUCT SPACES 


\title{
TOPOLOGICAL EXTENSIONS OF PRODUCT SPACES
}

\author{
FranK KosT
}

\begin{abstract}
The Stone-Čech compactification $\beta X$ and the Hewitt real-compactification $v X[6]$ of a completely regular $T_{1}$-space $X$ can be obtained as certain spaces of ultrafilters from the collection of zero sets of members of $C^{*}(X)[4]$. With the appropriate structure $\beta X$ is the space of all ultrafilters and $v X$ those with the countable intersection property. In this framework we give a necessary and sufficient condition for $\beta X \times$ $\beta Y \approx \beta(X \times Y)$.
\end{abstract}

Glicksberg [5], and then Frolík [3], established for infinite spaces $X$ and $Y$ that $\beta X \times \beta Y \approx \beta(X \times Y)$ if and only if $X \times Y$ is pseudocompact. Our condition is in terms of the zero sets of $X \times Y$ and we do not insist that $X$ and $Y$ be infinite. This result extends to arbitrary products. We give some sufficient conditions for $v X \times v Y \approx v(X \times Y)$ and in case $v X \times v Y($ or $v(X \times Y))$ is Lindelöf give a condition that is both sufficient and necessary.

1. For $Z$ a normal base [2] for the closed sets of $X$ and $F \in Z$ define $F^{*} \equiv\{$ ultrafilters from $Z$ that contain $F\} .\left\{F^{*}: F \in Z\right\}$ is a base for the closed sets of the ultrafilter space $\omega(Z)$ which is a Hausdorff compactification of $X$. The normality property of $Z$ is not needed to construct the $T_{1}$-compact space $\omega(Z)$. However, $\omega(Z)$ is a Hausdorff space if and only if $Z$ is a normal family. If $Z$ is the zero sets from $X$ then $\omega(Z) \approx \beta X$. Extensions of this kind are called Wallman-type. Say a base $Z_{1}$ separates a base $Z_{2}$ if disjoint members of $Z_{2}$ are contained in disjoint members of $Z_{1}$.

THEOREM 1.1. Let $Z_{1} \subset Z_{2}$ be normal bases for $X$. Then $\omega\left(Z_{1}\right) \approx \omega\left(Z_{2}\right)$ if and only if $Z_{1}$ separates $Z_{2}$.

Let $Z_{1}$ and $Z_{2}$ be normal bases for the closed sets of $X$ and $Y$.

THEOREM 1.2. $\omega\left(Z_{1}\right) \times \omega\left(Z_{2}\right)$ is a Wallman-type compactification of $X \times Y$.

Proof (Sketch). Let $Z_{1} \times Z_{2}=\left\{F \times G: F \in Z_{1}, G \in Z_{2}\right\}$ and $Z_{1} \times$ $Z_{2_{\Sigma}}$ be all finite unions from $Z_{1} \times Z_{2} . Z_{1} \times Z_{2_{\Sigma}}$ is the needed normal base, i.e., $\omega\left(Z_{1}\right) \times \omega\left(Z_{2}\right) \approx \omega\left(Z_{1} \times Z_{2_{\Sigma}}\right)$. The mapping $(\mathbb{Q}, \mathscr{B}) \rightarrow \mathbb{Q} \times \mathscr{B}$ 
is one-one from $\omega\left(Z_{1}\right) \times \omega\left(Z_{2}\right)$ onto the ultrafilters from $Z_{1} \times Z_{2}$ which are in one-one correspondence with those from $Z_{1} \times Z_{2_{\Sigma}}$. We take $(Q, \Re)$ $\rightarrow$ the ultrafilter from $Z_{1} \times Z_{2_{\Sigma}}$ that contains $\mathscr{Q} \times \mathscr{B}$. This is a homeomorphism. $\beta X \times \beta Y$ is, then, a Wallman-compactification of $X \times Y$.

Let $Z_{1}$ be the zero sets from $X$ and $Z_{2}$ those from $Y$. Denote the zero sets from $X \times Y$ by $Z(X \times Y)$. It is evident that $Z_{1} \times Z_{2_{\Sigma}} \subset Z(X \times Y)$.

Our main result is

THEOREM 1.3. $\beta X \times \beta Y \approx \beta(X \times Y)$ if and only if $Z_{1} \times Z_{2_{\Sigma}}$ separates the zero sets of $X \times Y$.

Proof. Assume that $Z_{1} \times Z_{2_{\Sigma}}$ separates $Z(X \times Y)$. By Theorem 1.1, $\omega\left(Z_{1} \times Z_{2_{\Sigma}}\right) \approx \beta(X \times Y)$. Using Theorem 1.2 we have $\beta X \times \beta Y \approx$ $\beta(X \times Y)$.

If $\beta X \times \beta Y \approx \beta(X \times Y)$ then Theorem 1.2 implies that $\omega\left(Z_{1} \times Z_{2_{\Sigma}}\right)$ $\approx \omega(Z(X \times Y))$ and by Theorem 1.1, $Z_{1} \times Z_{2_{\Sigma}}$ separates $Z(X \times Y)$.

Let $N$ be the positive integers with the discrete topology. In $N \times N$, $F_{1}=$ all points below the diagonal and $F_{2}=$ all points above the diagonal belong to $Z(N \times N)$ but cannot be separated by $Z_{1} \times Z_{2_{\Sigma}}$. In $R \times R$, where $R$ is the real line, $Z_{1} \times Z_{2_{\Sigma}}$ fails to separate the $y$-axis and $y=1 / x$.

REMARK. From Theorem 1.3 and Theorem 1 of [5] it is seen that, for $X$ and $Y$ infinite spaces, $X \times Y$ is pseudocompact if and only if $Z_{1} \times Z_{2_{\Sigma}}$ separates $Z(X \times Y)$.

Let $\left\{X_{\alpha}\right\}$ be a collection of completely regular $T_{1}$-spaces and $Z_{\alpha}$ the zero sets from $X_{\alpha}$.

THEOREM 1.4. I $\beta X_{\alpha}$ is a Wallman compactification of $\Pi X_{\alpha}$.

Proof (Sketch). Let $\Pi Z_{\alpha} \equiv\left\{\Pi F_{\alpha}: F_{\alpha} \in Z_{\alpha}, F_{\alpha}=X_{\alpha}\right.$ for all but finitely many $\alpha$ ) and $Z$ be all finite unions from $\Pi Z_{\alpha}$. $Z$ has sufficient properties to construct the compact $T_{1}$-space $\omega(Z)$. We show $\Pi \beta X_{\alpha} \approx \omega(Z)$ and it follows that $\omega(Z)$ is a Hausdorff space and that $Z$ is a normal base.

Remark. The Tychonoff Product Theorem can be obtained as a corollary to Theorem 1.4. In this case $\beta X_{\alpha} \approx X_{\alpha}$ and the homeomorphism gives $\Pi X_{\alpha}$ compact.

Let $Z$ be as above. Using Theorems 1.1 and 1.4 we arrive at an extension of our main result. 
THEOREM 1.5. $\Pi \beta X_{\alpha} \approx \beta\left(\Pi X_{\alpha}\right)$ if and only if $Z$ separates the zero sets of $\Pi X_{\alpha}$.

2. For $Z$ a normal base for $X$ let $p(Z)$ be the subspace of $\omega(Z)$ consisting of those points that have the countable intersection property (C.I.P.). $p(Z)$ is called a real-extension of $X$. Again, if $Z$ is the zero sets from $X$ then $p(X) \approx v X$. If $Z$ is a normal base, the family of countable intersections from $Z$, denoted $Z_{\cap}$, is a normal base and $p(Z) \approx p\left(Z_{\cap}\right)$. Although $Z_{\cap}$ may introduce "new" ultrafilters none of these will have the C.I.P. e.g. $Z=\{F \subset N: F$ or $N \backslash F$ is finite $\}$. $Z_{\cap}$ is all subsets of $N$ and $\omega\left(Z_{\cap}\right) \approx \beta N . \omega(Z)$ is the one-point compactification of $N$. Clearly $\omega\left(Z_{\cap}\right) \neq \omega(Z)$ yet $p\left(Z_{\cap}\right) \approx N \approx p(Z)$.

THEOREM 2.1. Let $Z_{1} \subset Z_{2}$ be normal bases for $X$ each closed under formation of countable intersections. In case $p\left(Z_{2}\right)\left(\right.$ or $\left.p\left(Z_{1}\right)\right)$ is Lindelöf it follows that $p\left(Z_{1}\right) \approx p\left(Z_{2}\right)$ if and only if $Z_{1}$ separates $Z_{2}$.

REMARK. We insist on the Lindelöf property to show the condition is necessary.

Let $Z_{1}$ and $Z_{2}$ be normal bases for $X$ and $Y$.

TheOREM 2.2. $p\left(Z_{1}\right) \times p\left(Z_{2}\right)$ is a real extension of $X \times Y$.

Proof (Sketch). $\omega\left(Z_{1}\right) \times \omega\left(Z_{2}\right) \approx \omega\left(Z_{1} \times Z_{2_{\Sigma}}\right)$ by Theorem 1.2. Under the mapping the image of $(Q, \Re)$ has the C.I.P. if and only if both $\mathbb{Q}$ and $\mathscr{B}$ do. Therefore $p\left(Z_{1}\right) \times p\left(Z_{2}\right) \approx p\left(Z_{1} \times Z_{2_{\Sigma}}\right)$.

Let $Z_{1}, Z_{2}$ be the zero sets of $X, Y$.

THEOREM 2.3. If $Z_{1} \times Z_{2_{\Sigma}}$ separates $Z(X \times Y)$ then $v X \times v Y \approx$ $v(X \times Y)$.

Proof. $\omega\left(Z_{1} \times Z_{2_{\Sigma}}\right) \approx \omega(Z(X \times Y))$ by Theorem 1.1.

If follows that $p\left(Z_{1} \times Z_{2_{\Sigma}}\right) \approx v(X \times Y)$. We have $v X \times v Y \approx$ $v(X \times Y)$ from Theorem 2.2.

TheOReM 2.4. Assume that $v X \times v Y($ or $v(X \times Y))$ is Lindelöf. Then $v X \times v Y \approx v(X \times Y)$ if and only if $Z_{1} \times Z_{2_{\Sigma_{n}}}$ separates $Z(X \times Y)$.

Proof. Note that $Z_{1} \times Z_{2_{\Sigma_{n}}} \subset Z(X \times Y)$. Theorems 2.1 and 2.2 establish sufficiency. 
If $v X \times v Y \approx v(X \times Y)$ then $p\left(Z_{1} \times Z_{2_{\Sigma}}\right) \approx p(Z(X \times Y))$ by Theorem 2.2 and the remarks preceding Theorem 2.1. From Theorem 2.1 we have $Z_{1} \times Z_{2_{\Sigma_{n}}}$ separates $Z(X \times Y)$.

There certainly are spaces $X, Y$ with $v X \times v Y$ Lindelöf and $v X \times v Y$ $\not(X \times Y)$. Take a pseudocompact space $X$ [4] with $X \times X$ not pseudocompact. $v X \times v X$ is compact, hence Lindelöf. However $v(X \times X)$ is not compact.

\section{REFERENCES}

[1] E. Čech, On bicompact spaces, Ann. of Math., 38 (1937), 823-844.

[2] O. Frink, Compactifications and semi-normal spaces, Amer. J. Math., 86 (1964), 602-607.

[3] Z. Frolik, The topological product of two pseudocompact spaces, Czechoslovak Math. J., 10 (1960), 339-349.

[4] L. Gillman and M. Jerison, Rings of Continuous Functions, Princeton, 1960.

[5] I. Glicksberg, Stone-Čech compactifications of products, Trans. Amer. Math. Soc., 90 (1959), 369-382.

[6] E. Hewitt, Rings of real-valued continuous functions I, Trans. Amer. Math. Soc., 64 (1948), 45-99.

[7] F. Kost, Finite products of Wallman spaces, Duke Math. J., (1971).

[8] M. H. Stone, Applications of the theory of Boolean rings to general topology, Trans. Amer. Math. Soc., 41 (1937), 375-481.

Received February 8, 1982.

SUNY

ONEONTA, NY 13820 


\section{PACIFIC JOURNAL OF MATHEMATICS EDITORS}

Donald BABBITT (Managing Editor)

University of California

Los Angeles, CA 90024

Hugo Rossi

University of Utah

Salt Lake City, UT 84112

C. C. Moore and Arthur Ogus

University of California

Berkeley, CA 94720
J. DugundiI

Department of Mathematics

University of Southern California

Los Angeles, CA 90089-1113

R. Finn and H. SAMELSON

Stanford University

Stanford, CA 94305

ASSOCIATE EDITORS
R. ARENS
E. F. BECKENBACH
B. H. NeUmanN
F. WOLF
K. YosHIDA (1906-1982)

\section{SUPPORTING INSTITUTIONS}

UNIVERSITY OF ARIZONA

UNIVERSITY OF BRITISH COLUMBIA

CALIFORNIA INSTITUTE OF TECHNOLOGY

UNIVERSITY OF CALIFORNIA

MONTANA STATE UNIVERSITY

UNIVERSITY OF NEVADA, RENO

NEW MEXICO STATE UNIVERSITY

OREGON STATE UNIVERSITY
UNIVERSITY OF OREGON

UNIVERSITY OF SOUTHERN CALIFORNIA

STANFORD UNIVERSITY

UNIVERSITY OF HAWAII

UNIVERSITY OF TOKYO

UNIVERSITY OF UTAH

WASHINGTON STATE UNIVERSITY

UNIVERSITY OF WASHINGTON 


\section{Pacific Journal of Mathematics}

\section{Vol. 108, No. $1 \quad$ March, 1983}

Waleed A. Al-Salam and A. Verma, $q$-Konhauser polynomials $\ldots \ldots \ldots \ldots 1$

Alfred David Andrew, The Banach space JT is primary $\ldots \ldots \ldots \ldots \ldots . . .6$

Thomas E. Bengtson, Bessel functions on $P_{n} \ldots \ldots \ldots \ldots \ldots \ldots$

Joaquim Bruna Floris and Francesc Tugores, Free interpolation for

holomorphic functions regular to the boundary $\ldots \ldots \ldots \ldots \ldots \ldots \ldots \ldots$

Peter Dierolf and Susanne Dierolf, Topological properties of the dual pair

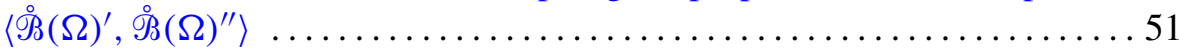

Gerald Arthur Edgar, An ordering for the Banach spaces $\ldots \ldots \ldots \ldots \ldots 83$

Basil Gordon, A proof of the Bender-Knuth conjecture . . . . . . . . . . . . . 99

Harold T. Hodes, A minimal upper bound on a sequence of Turing degrees

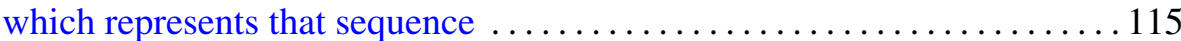

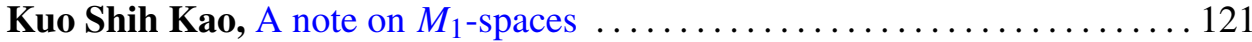

Frank Kost, Topological extensions of product spaces ................ 129

Eva Lowen-Colebunders, On the convergence of closed and compact

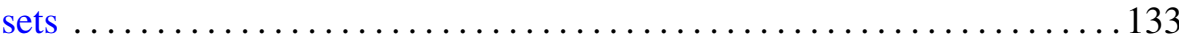

Doron Lubinsky, Divergence of complex rational approximations . . . . . . 141

Warren May and Elias Hanna Toubassi, Endomorphisms of rank one

mixed modules over discrete valuation rings $\ldots \ldots \ldots \ldots \ldots \ldots \ldots \ldots \ldots$

Richard Patrick Morton, The quadratic number fields with cyclic

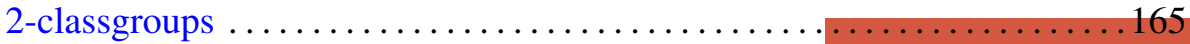

Roderic Murufas, Rank of positive matrix measures . . . . . . . . . . . . 177

Helga Schirmer, Fixed point sets of homotopies . . . . . . . . . . . . . 191

E. Taflin, Analytic linearization of the Korteweg-de Vries equation ........ 203

James Thomas Vance, Jr., $L^{p}$-boundedness of the multiple Hilbert

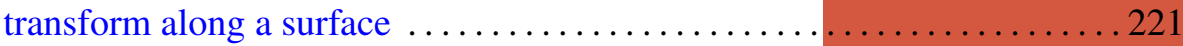

Hiroshi Yamaguchi, A property of some Fourier-Stieltjes transforms . . . . . 243 